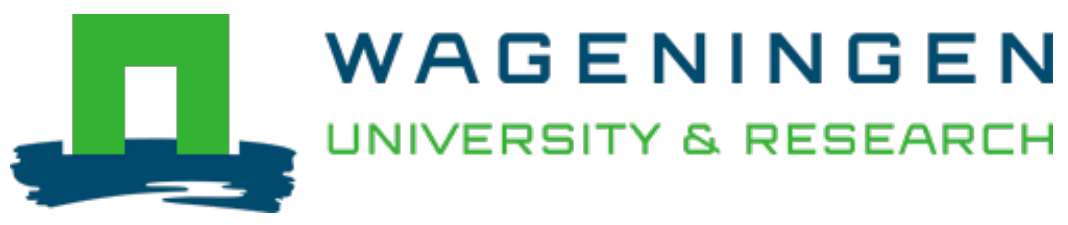

\title{
Selective Positioning of Nanosized Metal-Organic Framework Particles at Patterned Substrate Surfaces
}

\section{Chemistry of materials}

Semrau, Anna Lisa; Pujari, Sidharam P.; Stanley, Philip Matthew; Wannapaiboon, Suttipong; Albada, Bauke et al

https://doi.org/10.1021/acs.chemmater.0c02871

This publication is made publicly available in the institutional repository of Wageningen University and Research, under the terms of article $25 \mathrm{fa}$ of the Dutch Copyright Act, also known as the Amendment Taverne. This has been done with explicit consent by the author.

Article 25 fa states that the author of a short scientific work funded either wholly or partially by Dutch public funds is entitled to make that work publicly available for no consideration following a reasonable period of time after the work was first published, provided that clear reference is made to the source of the first publication of the work.

This publication is distributed under The Association of Universities in the Netherlands (VSNU) 'Article $25 \mathrm{fa}$ implementation' project. In this project research outputs of researchers employed by Dutch Universities that comply with the legal requirements of Article $25 \mathrm{fa}$ of the Dutch Copyright Act are distributed online and free of cost or other barriers in institutional repositories. Research outputs are distributed six months after their first online publication in the original published version and with proper attribution to the source of the original publication.

You are permitted to download and use the publication for personal purposes. All rights remain with the author(s) and / or copyright owner(s) of this work. Any use of the publication or parts of it other than authorised under article $25 \mathrm{fa}$ of the Dutch Copyright act is prohibited. Wageningen University \& Research and the author(s) of this publication shall not be held responsible or liable for any damages resulting from your (re)use of this publication.

For questions regarding the public availability of this publication please contact openscience.library@wur.nl 


\section{Selective Positioning of Nanosized Metal-Organic Framework Particles at Patterned Substrate Surfaces}

Anna Lisa Semrau, Sidharam P. Pujari, Philip Matthew Stanley, Suttipong Wannapaiboon, Bauke Albada, Han Zuilhof, and Roland A. Fischer*

Cite This: Chem. Mater. 2020, 32, 9954-9963

Read Online

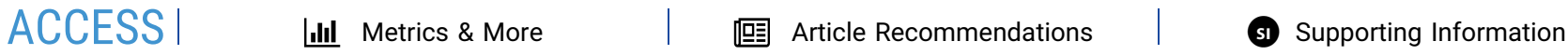

ABSTRACT: Herein, we describe the selective positioning of metal-organic framework (MOF) nanoparticles UiO-66 (Universitet i Oslo; $\mathrm{Zr}_{6} \mathrm{O}_{4}(\mathrm{OH})_{4}(\mathrm{bdc})_{6} ; \mathrm{bdc}^{2-}=$ benzene-1,4dicarboxylate) and MIL-101 (Matérial Institut Lavoisier, $\mathrm{Cr}_{3} \mathrm{O}$ $\left.(\mathrm{OH})\left(\mathrm{H}_{2} \mathrm{O}\right)_{2}(\mathrm{bdc})_{3}\right)$ at defined positions on a patterned substrate. For this purpose, patterned alkyne- and carboxylic acid-terminated self-assembled organic monolayer (SAM)-modified silicon surfaces were prepared by liquid immersion and microcontact printing $(\mu \mathrm{CP})$. Preformed UiO-66 and MIL-101 nanometer-sized MOFs (NMOFs) were synthesized by solvothermal synthesis, and the nanocrystallite particles' exterior surface was functionalized in order to generate reactive sites (such as azides

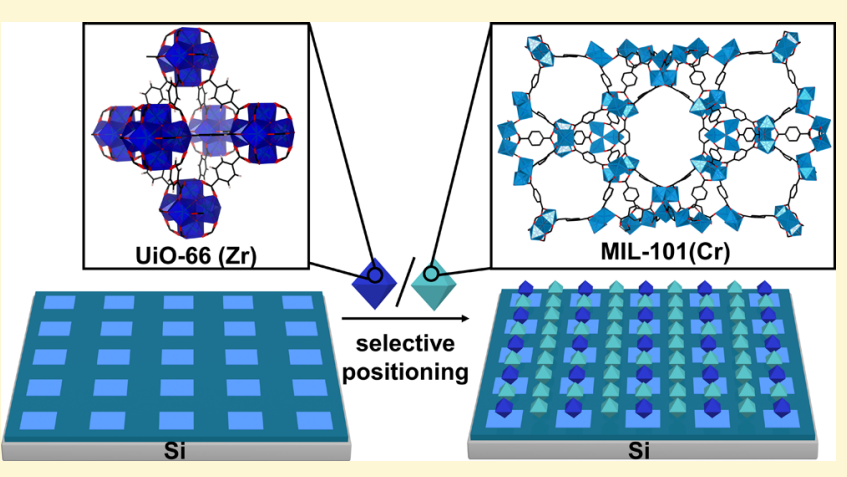
and amines) at the NMOFs. Copper-catalyzed alkyne azide cycloaddition and $\mathrm{N}$-hydroxysuccinimide-mediated amide formation were used to selectively position the NMOFs at the surface of pre-patterned substrates. The resulting surfaces were thoroughly investigated by scanning electron microscopy, infrared spectroscopy, and X-ray photoelectron spectroscopy, confirming the validity of the presented approach. We hope that our research paves the way for microsystem integration of NMOFs, for example, in microfluidic devices/reactors, and further investigation of their enhanced catalytic activity.

\section{INTRODUCTION}

Surface engineering-the alteration of surfaces to target specific properties that could not be achieved by the substrate itself-is a useful tool in material science. The process can render the resulting composite materials catalytically active, ${ }^{1,2}$ introduce optical properties, ${ }^{3,4}$ or provide protection from degradation. ${ }^{5}$

A similar variety of fascinating properties have been reported for metal-organic frameworks (MOFs), which can be ascribed to their large accessible surface area, uniform and tunable pore size, and chemical modularity. ${ }^{6}$ MOFs are explored for applications in gas adsorption and separation, ${ }^{7}$ heterogeneous catalysis, ${ }^{8}$ sensing platforms, ${ }^{9}$ biosciences, ${ }^{10}$ and optical devices. ${ }^{11}$ The immobilization of MOFs onto surfacesessentially surface engineering-can potentially enhance their properties (because of the reduction of grain boundaries) and enable their integration into devices. Especially relevant for this goal is control over the spatial arrangement of the immobilized MOFs, for example, as catalysts in tandem, cascade, or vectorial catalysis.

In the past, MOFs were selectively grown (bottom-up approach) on patterned substrates, which has been shown by Ocal et al. ${ }^{12}$ and Falcaro et al. ${ }^{13}$ among others, ${ }^{14}$ but (topdown) immobilization of presynthesized MOF particles on patterned surfaces has to the best of our knowledge not been reported, yet. Because of the high control over the preformed particle shape and size, the top-down approach offers a distinct advantage. Different groups reported that the catalytic activity of the particles increases with decreasing particle diameter. ${ }^{15,16}$ Unfortunately, like other nanoparticles, NMOFs tend to agglomerate especially during catalysis. ${ }^{17}$ This process is accompanied by decreasing catalytic activity. We showed that this process can be prevented by the immobilization of the NMOFs on surfaces. ${ }^{17}$ Additionally, we showed that surfaceanchored nanometer-sized MOFs (SA-NMOFs) exhibit substantially enhanced catalytic activities as compared to bottom-up MOF thin films which we ascribe to lower diffusion barriers and/or reducing diffusion limitations. ${ }^{17}$

Our goal of combining different reactive NMOFs in a microfluidic device in a controlled fashion as a tangible application for novel surface engineering methods urges us to

Received: July 9, 2020

Revised: November 10, 2020

Published: November 25, 2020 
(a)
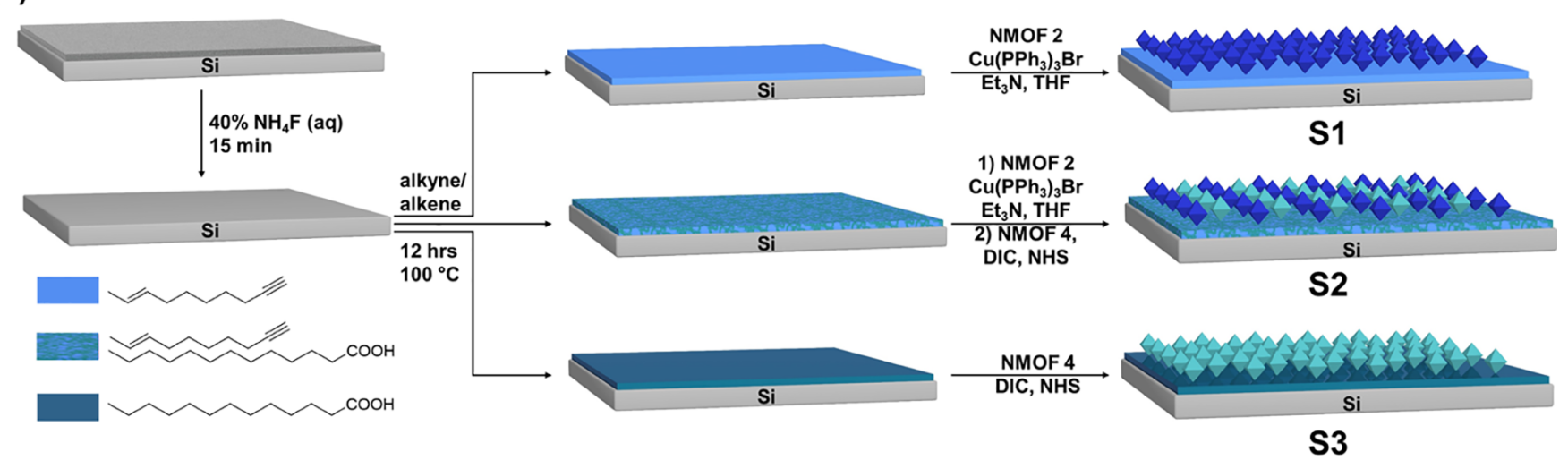

\section{(b)}

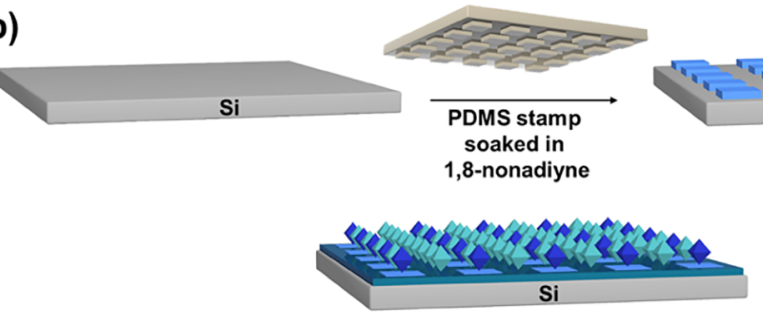

S5

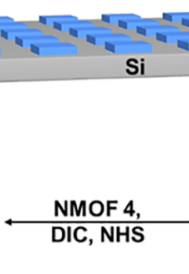

DIC, NHS

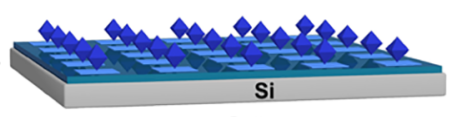

backfilling 10-undecenoic acid

S4

Figure 1. Schematic approach to selectively position NMOF 2 and 4 on silicon substrates. (a) In the first step, silicon substrates are treated with a $40 \%$ aqueous $\mathrm{NH}_{4} \mathrm{~F}$ solution to remove the native oxide layer. This step is followed by a reaction of the silicon substrates in neat 1,8 -nonadiyne, 10 undecenoic acid, or a 1:1 solution of 1,8-nonadiyne and 10-undecenoic acid. Finally, the functionalized silicon surfaces are reacted with NMOF 2 or 4 or both, respectively, to form S1-3. (b) Selective spatial distribution of the NMOFs 2 and 4 was shown by surface patterning via microcontact printing on a silicon surface. Therefore, an etched silicon substrate was contacted with a PDMS stamp, which was soaked in neat 1,8-nonadiyne. The backfilling procedure was performed for $12 \mathrm{~h}$ at room temperature with 10 -undecenoic acid. The patterned silicon surface was sequentially reacted with NMOF 2 (S4) and NMOF 4 (S5).

develop the surface chemistry of NMOFs and their areaselective substrate positioning in some detail.

For this approach, we used the NMOFs UiO- $66^{18}$ (Universitet i Oslo; $\mathrm{Zr}_{6} \mathrm{O}_{4}(\mathrm{OH})_{4}(\mathrm{bdc})_{6} ; \mathrm{bdc}^{2-}=1$,4-benzene dicarboxylate) and MIL-101 ${ }^{19}$ (Matérial Institut Lavoisier, $\left.\mathrm{Cr}_{3} \mathrm{O}(\mathrm{OH})\left(\mathrm{H}_{2} \mathrm{O}\right)_{2}(\mathrm{bdc})_{3}\right)$ because both compounds are wellknown for their physical and chemical stability. ${ }^{20-22}$ Additionally, their crystallization behavior has been thoroughly investigated and therefore literature reporting on the synthesis of nanometer-sized crystals is available. ${ }^{23,24}$ In order to immobilize the NMOFs by the amide bond formation and copper-catalyzed azide-alkyne cycloaddition (CuAAC) reaction, an introduction of functional groups (namely, azide and amino groups) on the exterior surface of the NMOF was necessary. These functionalities were introduced to the outer surface of the NMOFs by the formation of amide bonds between dangling carboxylic acid groups of bdc linkers and $\omega$ functionalized alkane- $\alpha$-amines ( $\omega$-amino or $\omega$-azido). Moreover, the surface functionalization by the immersion of the $\mathrm{NH}_{4}$ F-etched Si slides in neat alkyne or alkene solutions at 100 ${ }^{\circ} \mathrm{C}$ overnight (see Figure 1a). Hence, the substrate surfaces were modified by a self-assembled organic monolayer (SAM) possessing the alkyne, carboxylic acid, or mixed terminated groups at the surface. Additionally, we prepared some patterned surfaces (see Figure $1 \mathrm{~b}$ ) via microcontact printing $(\mu \mathrm{CP})$, that is, by treating the etched surfaces with a PDMS stamp in a glovebox $\left(\mathrm{O}_{2}<0.1 \mathrm{ppm}\right.$, and $\left.\mathrm{H}_{2} \mathrm{O}<0.1 \mathrm{ppm}\right)$ overnight, and additional backfilling afterward yielding a patterned surface consisting of specific areas with alkyne and areas with carboxylic acid termination. In a second step, we reacted these surfaces with surface-functionalized NMOFs. UiO-66 (NMOF 1) or MIL-101 (NMOF 3) nanoparticles were therefore functionalized on their nanocrystallite particles' exterior surface forming azide-functionalized UiO-66 (NMOF 2) or amine-functionalized MIL-101 (NMOF 4). Then, these moieties were exploited for the selective immobilization of the NMOFs at the functionalized $\mathrm{Si}$ surfaces. The alkyneterminated Si surface (alkyne, mixed, and patterned surface) was reacted with NMOF 2 catalyzed by a $\mathrm{Cu}(\mathrm{I})$ catalyst. In a second step, the carboxylic acid-terminated $\mathrm{Si}$ surface (carboxylic acid, mixed, and patterned surface) was reacted with NMOF 4. The amide bond formation is facilitated by diisopropyl carbodiimide (DIC) and $N$-hydroxysuccinimide (NHS). We used several techniques such as scanning electron microscopy (SEM), X-ray photoelectron spectroscopy (XPS), infrared reflection absorption spectroscopy (IRRAS), attenuated total reflectance infrared spectroscopy (IR-ATR), dynamic light scattering (DLS), powder X-ray diffraction (PXRD), and nitrogen adsorption experiments (BET) to demonstrate the properties of the synthesized NMOFs and to show that they were successfully and selectively positioned.

\section{RESULTS AND DISCUSSION}

Substrates: Functionalization of Silicon Substrates with Monolayers of 1,8-Nonadiyne and 10-Undecenoic Acid and with Patterned Mixed Monolayers. Alkyne- and carboxylic acid-terminated monolayers and mixed monolayers on silicon surfaces were formed by the reaction of $\mathrm{NH}_{4} \mathrm{~F}$ etched silicon substrates with neat 1,8-nonadiyne, 10undecanoic acid, and a 1:1 $\mathrm{M}$ ratio mixture of both chemicals, 

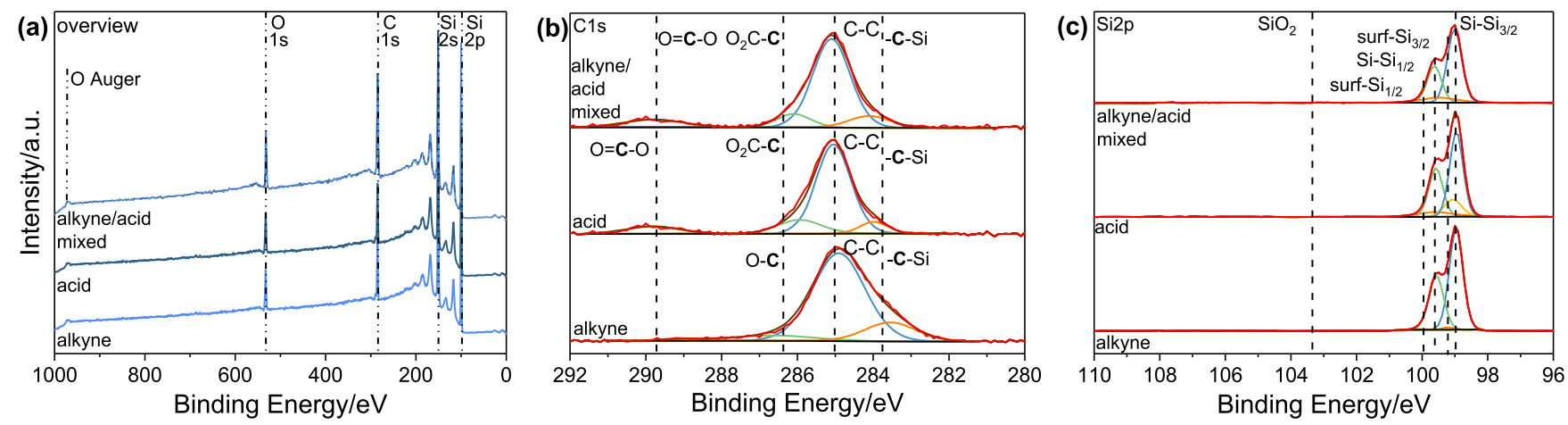

Figure 2. XPS survey scan (a) of alkyne, acid, and alkyne/acid-terminated monolayers and C 1s (b) and Si 2p (c) XPS narrow scans.

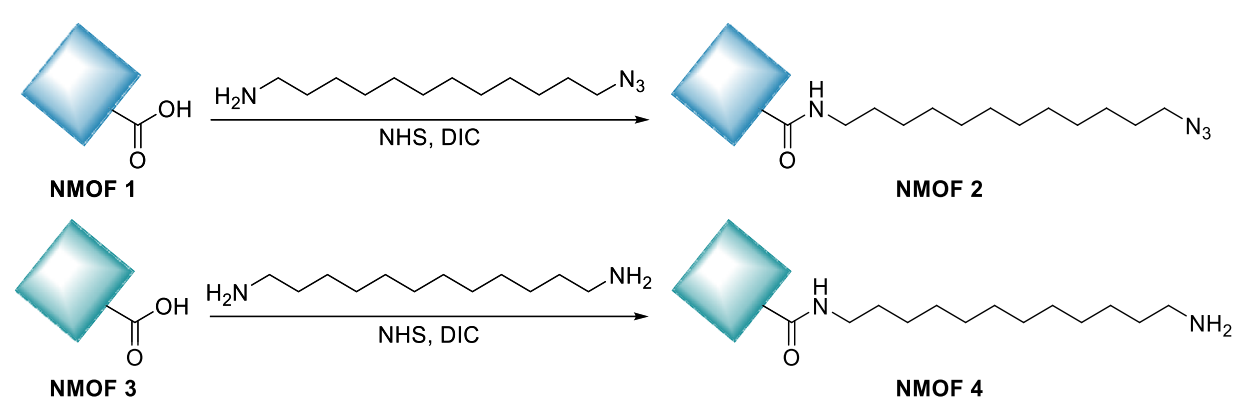

Figure 3. UiO-66 (NMOF 1) and MIL-101 (NMOF 3) are reacted with ADA and DAD, respectively, to form functionalized MOF NPs NMOF 2 and NMOF 4.

Table 1. Particle Diameters, Hydrodynamic Diameters, and Crystallite Domain Sizes of NMOF 1-4 Determined by SEM, DLS, and PXRD.

\begin{tabular}{|c|c|c|c|c|}
\hline determined by & SEM & PXRD & & DLS \\
\hline Sample & particle diameter $[\mathrm{nm}]$ & crystallite domain size $[\mathrm{nm}]$ & hydrodynamic diameter $[\mathrm{nm}]$ & hydrodynamic diameter $[\mathrm{nm}]$ after 4 weeks \\
\hline NMOF 1 & $21.0 \pm 3.3$ & $17.7 \pm 2.1$ & $164 \pm 24$ & $531 \pm 76$ \\
\hline NMOF 2 & $23.4 \pm 2.5$ & $16.9 \pm 1.5$ & $91 \pm 38$ & $78 \pm 24$ \\
\hline NMOF 3 & $26.7 \pm 3.9$ & $12.4 \pm 2.8$ & $79 \pm 33$ & $106 \pm 47$ \\
\hline NMOF 4 & $24.6 \pm 3.8$ & $13.0 \pm 2.3$ & $91 \pm 34$ & $91 \pm 46$ \\
\hline
\end{tabular}

respectively. The resulting surfaces were investigated by XPS, IRRAS, and contact angle measurements.

XPS spectra of the three modified surfaces show the presence of $\mathrm{Si}, \mathrm{C}$, and $\mathrm{O}$ as previously reported in the literature (see Figure 2). ${ }^{25,26}$ In the case of 1,8-nonadiyne, the oxygen content can be explained by covalently bound oxygen (C-O), which presumably originates from the 1,8 -nonadiyne. ${ }^{26}$ The XPS C 1s narrow scan can be deconvoluted into different components which can be assigned to a $\mathrm{C}-\mathrm{C}$ bond peak $(285.0 \mathrm{eV}), \mathrm{Si}-\mathrm{C}=\mathrm{C}$ bonds $(284.0 \mathrm{eV}), \mathrm{C}-\mathrm{O} / \mathrm{C}-\mathrm{CO}_{2} \mathrm{H}$ $(286.0 \mathrm{eV})$, and $\mathrm{C}-\mathrm{CO}_{2} \mathrm{H}(290.0 \mathrm{eV}) .^{25-27}$ A glance at the $\mathrm{Si}$ $2 \mathrm{p}$ narrow scan reveals that the $\mathrm{Si}-\mathrm{O}$ peak at $101-104 \mathrm{eV}$ is nonexistent. This observation indicates the formation of densely packed SAMs that prevented the oxidation of the etched $\mathrm{Si}-\mathrm{H}$ substrate surfaces. The $\mathrm{O}$ 1s narrow scan can be found in the Supporting Information, Chapter S3. IRRAS spectra (see Supporting Information, Chapter S4) show the symmetric and antisymmetric $\mathrm{C}-\mathrm{H}$ stretch vibrations and the $\mathrm{C}=\mathrm{O}$ stretching vibration for the carboxylic acid-terminated self-assembled monolayer, in accordance with the literature. $^{28,29}$ The static water contact angle of the surfaces (see Supporting Information, Chapter S5) is $93 \pm 3^{\circ}$ for the alkyneterminated monolayer, ${ }^{26,27} 65^{\circ}$ for the $-\mathrm{COOH}$-terminated monolayers, ${ }^{30}$ and $54^{\circ}$ for the native oxide surface on silicon. Therefore, IRRAS spectra, XPS spectra, and water contact angle measurements confirm the successful formation of dense monolayers on the etched silicon surfaces.

NMOFs: Synthesis and Outer Surface Functionalization. Nanometer-sized UiO-66 (NMOF 1) and MIL-101 (NMOF 3) particles were synthesized via two different approaches. For UiO-66 nanoparticles (NPs), we chose a typical solvothermal synthesis with a high concentration of reactants, a modulator, and a low reaction temperature, which resulted in a white, colloidal UiO-66 solution. ${ }^{23}$ MIL-101 NPs were synthesized under harsh reaction conditions $\left(\mathrm{H}_{2} \mathrm{O}, 200\right.$ $\left.{ }^{\circ} \mathrm{C}, 1 \mathrm{~min}\right)$ in a microwave reactor, ${ }^{24}$ resulting in a green colloidal solution of MIL-101. The correlation between these parameters and the particle size was recently published, together with an investigation of the influence of acidity, the concentration of the ligand, and modulator on the particle size. $^{31}$

As shown in Figure 3, NMOF 1 and NMOF 3 are functionalized on their outer surface. Therefore, the carboxylic acid groups of the dangling bdc linkers on the outer surface of the NMOFs are utilized and reacted with 12-azido dodecane1-amine (ADA) or 1,12-diamino dodecane (DAD), respectively, to form the functionalized NMOFs 2 and 4 . Note that, the surface functionalization not only stabilizes the NMOFs but also provides additional functional groups on the outer surface. NMOFs 1-4 were further characterized by SEM, 

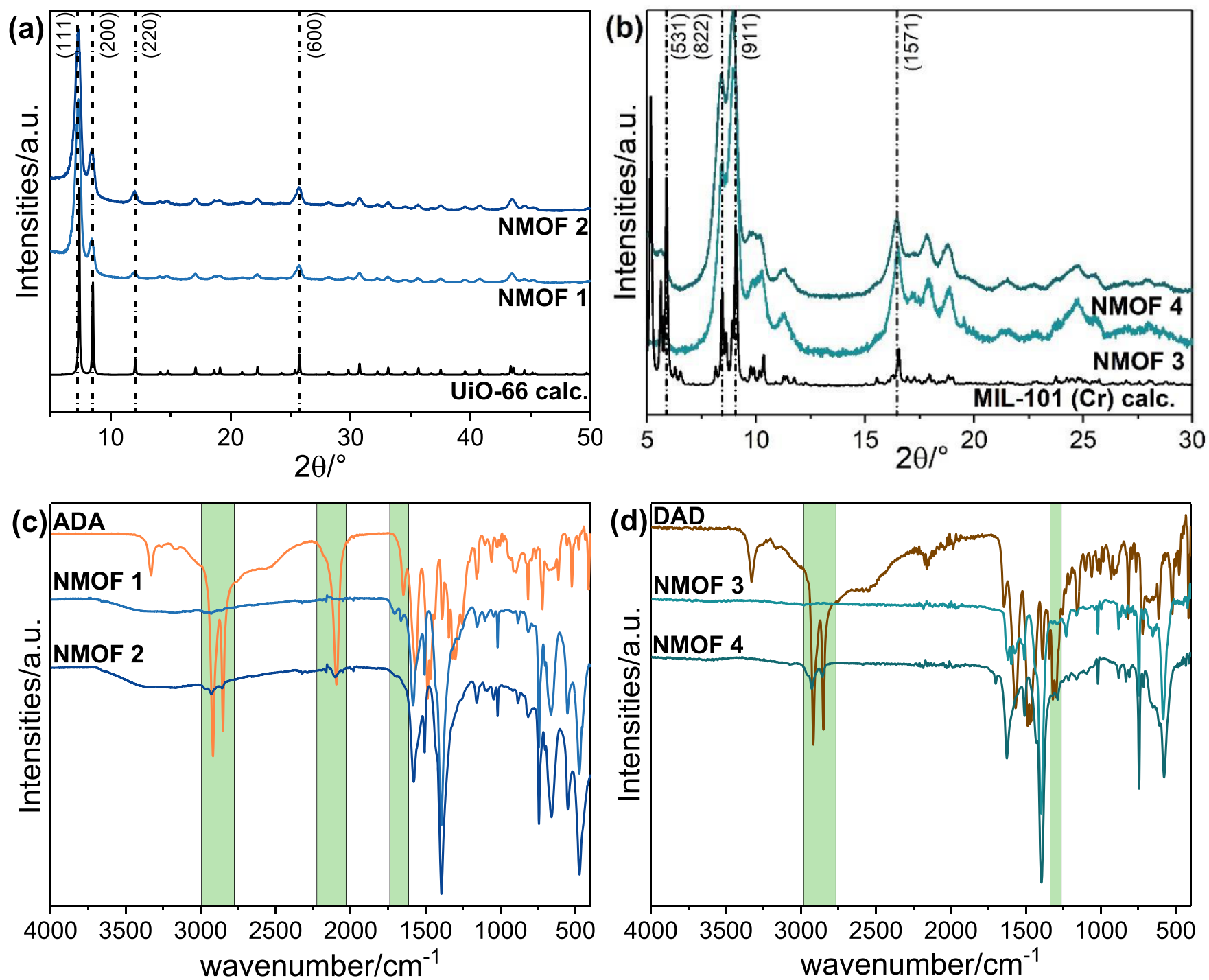

Figure 4. PXRD diffractograms of the activated $\left(100^{\circ} \mathrm{C}, 12 \mathrm{~h}\right)$ NMOFs 1-2 (light/dark blue) (a) and NMOF 3-4 (light/dark turquoise) (b). The PXRD reference patterns (black) were calculated with Vesta from the single crystal structure of UiO-66 (CCDC 733458) and MIL-101 (Cr) (CCDC 605510), respectively. The IR spectra of NMOFs 1-2 and ADA (orange) and NMOF 3-4 and DAD (brown) are shown in (c) and (d). Specific areas of interest are highlighted in green.

PXRD, DLS, XPS, IR, TGA, BET, and NMR following NP digestion. The particle diameter, hydrodynamic diameter, and crystallite domain size were determined by SEM, DLS, and PXRD, respectively, and are shown in Table 1.

The particle diameter determined by SEM (see Supporting Information, Chapter S6) is about $\sim 23 \mathrm{~nm}$ for all NMOFs and changes slightly by surface functionalization. The values for NMOF 1 and NMOF 3 were already reported in the literature [22 nm $\pm 8 \mathrm{~nm}$ (NMOF 1) and ${ }^{23} 22 \mathrm{~nm} \pm 5 \mathrm{~nm}$ (NMOF $3)^{24}$ and are similar to our measurements. The crystalline domain size of the samples was determined with the Scherrer equation applied to multiple reflections (see Supporting Information, Chapter S7 for more information). The calculated domain sizes in comparison with the SEM data suggest that each of the particles contains 1-2 crystallite domains. DLS spectra (see Supporting Information, Chapter S8) show the average hydrodynamic diameter of the particles in solution, which is typically larger than the particle diameter because of the solvent shell adsorbed at the surface of the NMOF. The measured diameter of the NMOF shows that the NMOF 1 aggregates over time resulting in an increased hydrodynamic radius and precipitation of the particles from the solution. In contrast to this, NMOF 3 forms a more stable colloidal solution. A slight increase in the hydrodynamic radius is observed over time, but the solution is still colloidal, and any increase after 4 weeks is still within the margin of error. Hence, NMOF 2 and $\mathbf{4}$ are stable colloidal solutions, which do not aggregate over time.

The PXRD patterns of the NMOFs 1-4 (Figure 4) show that the synthesized NMOFs feature the targeted structure of bulk UiO-66 and MIL-101, respectively. Moreover, they indicate that the structure is retained during the particle functionalization process. Additionally, the IR spectra reveal that NMOFs 2 and $\mathbf{4}$ are composite materials consisting of $\mathrm{ADA}$ and NMOF 1 and DAD and NMOF 3, respectively. The $\mathrm{CH}_{2}$ stretching vibrations $\left(\sim 2900 \mathrm{~cm}^{-1}\right)$, which are absent in the spectra of NMOF 1 and 3 become visible in the spectra of NMOF 2 and 4. Additionally, the azide stretching vibration around $2100 \mathrm{~cm}^{-1}$ can be observed for NMOF 2 and the band at $1664 \mathrm{~cm}^{-1}$ which originates from the $\mathrm{OH}$ bending vibration 

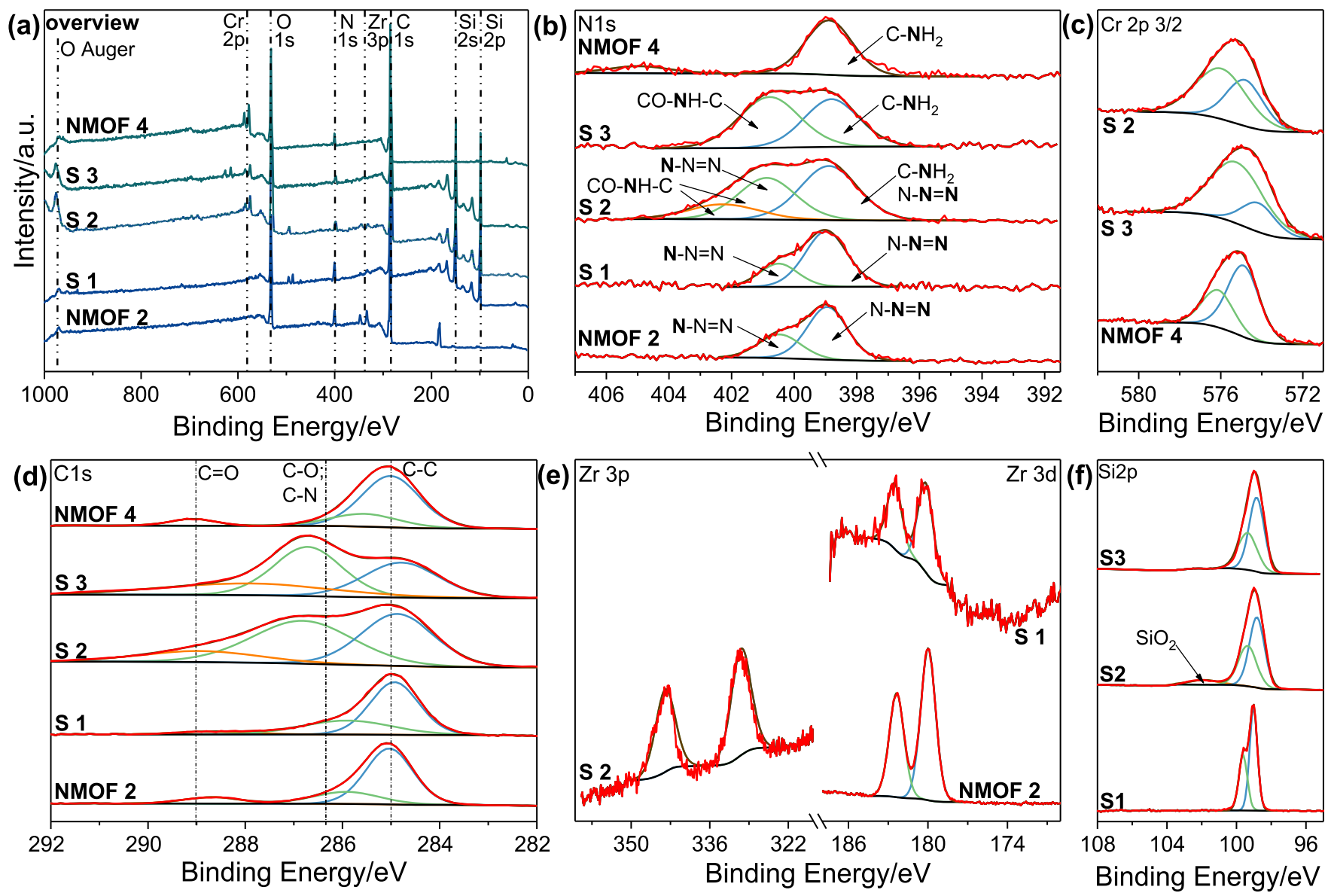

Figure 5. XPS survey spectra (a) of NMOFs 2 and 4 and S1-3 and the corresponding narrow scan spectra for N 1s (b), Cr 2 $\mathrm{p}_{3 / 2}$ (c), C 1s (d), Zr $3 \mathrm{p} / 3 \mathrm{~d}(\mathrm{e})$, and $\operatorname{Si} 2 \mathrm{p}(\mathrm{f})$.

of the free carboxylic acid is present in NMOF 1, but absent in NMOF 2. These observations confirm the successful surface functionalization of NMOF 1 and NMOF 3 to form NMOF 2 and 4 , respectively.

Another critical point was to determine the amount of $\mathrm{ADA}$ or $\mathrm{DAD}$ that was incorporated into the NMOF structure. Therefore, we digested the NMOFs 2 and $\mathbf{4}$ in a solution of DMSO- $\mathrm{d}_{6} / \mathrm{DCl}$ as described before and measured the NMR spectra. ${ }^{32}$ The full calculation of the functionalization, as well as the NMR spectra, are documented in the Supporting Information, Chapter S9. The obtained functionalization of NMOF 2 is $15.8 \mathrm{~mol} \%(11.3 \mathrm{wt} \%)$ and for NMOF 4, about $21.0 \mathrm{~mol} \%$ (14.9 wt \%). This again demonstrates the successful functionalization of the NMOF particles.

We investigated the accessible inner surface areas of the NMOF particle surface area before and after the exterior NMOF particle surface functionalization with nitrogen adsorption experiments. The $\mathrm{N}_{2}$ adsorption isotherms measured at $77 \mathrm{~K}$ are documented in the Supporting Information, Chapter S10. The obtained weight-specific BET surface areas show an expected slight reduction by $15.3 \%$ [NMOF $2\left(918 ; 777 \mathrm{~m}^{2} / \mathrm{g}\right)$ ] and 12.0\% [NMOF 4 (1856; $\left.\left.1632 \mathrm{~m}^{2} / \mathrm{g}\right)\right]$, respectively, which matches with the range of the weight-specific NMOF particle surface functionalization. Overall, the internal surface area of the functionalized NMOFs is still accessible and only slightly reduced.

Immobilization: Anchoring NMOFs at the Functionalized Surfaces. In the final step, the NMOFs 2 and 4 were immobilized on the matching surfaces. Therefore, NMOF 2 was reacted with the alkyne-terminated and alkyne/acid mixed surface in the CuAAC reaction forming covalent triazole linkages between the NMOFs and the functionalized surface. NMOF 4 and the carboxylic acid and alkyne/acid mixed surfaces were synthesized utilizing DIC and NHS to form amide bonds. The resulting surfaces NMOF 2@alkyne-Si (S1), NMOF 2 and 4@alkyne/acid mixed-Si (S2), and NMOF 4@ acid-Si (S3) were investigated by XPS and SEM.

XPS survey spectra (Figure 5) reveal the presence of N, O, and $\mathrm{C}$ in all samples as expected. Additionally, $\mathrm{Zr}$ is present in NMOF 2, S1, and S2, and Cr in NMOF 4, S3, and S2 also matching expectations. Moreover, Si can be found in S1-3 because of the immobilization onto a surface of a silicon substrate. The absence of a peak at $\sim 933 \mathrm{eV}\left(\mathrm{Cu} 2 \mathrm{p}_{3 / 2}\right)$ in the samples $\mathbf{S 1}$ and $\mathbf{S 2}$ indicates that there is no residual $\mathrm{Cu}(\mathrm{I})$ catalyst present on the substrate. The $\mathrm{C} 1 \mathrm{~s}$ and $\mathrm{N} 1 \mathrm{~s}$ XPS narrow scans are of particular interest. The $\mathrm{N} 1 \mathrm{~s}$ narrow scan of NMOF 2 and $\mathbf{S 1}$ can be fitted into two components at 400 and $401.6 \mathrm{eV}$, which can be assigned to $\mathrm{N}-\mathrm{N}=\mathrm{N}$ and $\mathrm{N}-\mathrm{N}=$ $\mathrm{N}$, respectively. ${ }^{33,34}$ The calculated stoichiometric ratio of $1: 2$ is in the range of the measured ratio of $1: 2.1$. The spectra of NMOF 4 and S3 display a major peak at $399.0 \mathrm{eV}$, which can be assigned to the amino group on the surface. $\mathbf{S} 3$ shows an additional peak at $400.8 \mathrm{eV}$, which can be assigned to the formed amide bonds. The S2 N1s narrow scan combines the $\mathrm{C}-\mathrm{NH}_{2}$ groups from NMOF 4 and the $\mathrm{N}_{3}$ groups from NMOF 2, which are partly free dangling groups and partly 


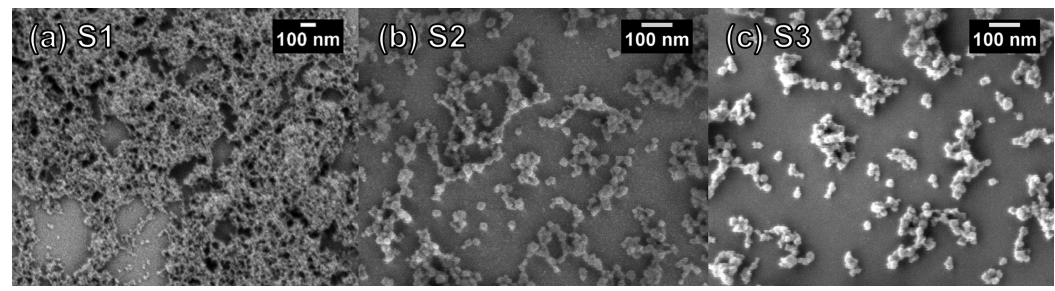

Figure 6. SEM images of S1-3 $(a-c)$. The additional SEM images and histograms of the size distributions can be found in the Supporting Information, Chapter S11.

involved in an amide or triazole bond to the surface resulting in a complicated spectrum. The C 1s spectra of NMOFs 2 and 4 and S1-3 can be assigned as follows: the major peak at 285.0 $\mathrm{eV}$ originates from $\mathrm{C}-\mathrm{C}$ bonds, either from the surface modification or from the bdc linker of the NMOF or the alkyl chains of the functionalization of the NMOF; the peak around $286.4 \mathrm{eV}$ stems from $\mathrm{C}$ bound to electronegative atoms such as $\mathrm{O}$ or $\mathrm{N}$. This peak is more pronounced in the spectra of S1-3 because of the additional triazole and amide bonds, which are formed for the immobilization. Finally, the peak at $289.0 \mathrm{eV}$ can be assigned to $\mathrm{C}=\mathrm{O}$ species, which are either present in the carboxylic acid of the surface functionalization or at the bdc linker of the NMOFs. Overall, this proves the successful immobilization of NMOF 2 at the alkyne surface, NMOF 4 at the carboxylic acid surface, and both NMOFs at the mixed alkyne/acid surface.

Furthermore, we investigated the surfaces of the substrates by employing electron microscopy (Figure 6). These images should display the distribution and arrangement of the NMOFs on the surfaces. First, the images show small particles in the same size regime as reported and measured for the NMOFs. The particles are slightly agglomerated but only to a small extent, and the individual particles are still visible. A more detailed analysis of 200 particles, respectively, shows that the average particle diameter of the NMOFs is $18.0 \pm 2.8 \mathrm{~nm}$ (S1), $22.4 \pm 3.3 \mathrm{~nm}$ (S2), and $24.2 \pm 3.3 \mathrm{~nm}$ (S3), which agrees with the measured size of NMOF 2 and 4 . NMOF 2 seems to agglomerate more during the reaction. Therefore, we observed a denser particle structure in S1 than for NMOF 4 in S3.

Patterning: Anchoring different NMOFs at the Functionalized Surfaces. The final experiment was the immobilization of NMOFs 2 and 4 at a micro-patterned surface. For this reason, we used an etched Si substrate and prepared a PDMS stamp with a pattern of $20 \times 20 \mu \mathrm{m}$ elevations, which was soaked in neat 1,8 -nonadiyne and placed on an etched $\mathrm{Si}$ substrate at room temperature under inert conditions for $12 \mathrm{~h}$. Afterward, the stamp was removed and the surface was placed in a neat solution of 10-undecenoic acid for backfilling of the surface, to create a micro-pattern of alkyneand acid-terminated areas. This patterned surface was then consecutively reacted with NMOF 2 (S4) and NMOF 4 (S5). The resulting surfaces ( $\mathbf{S} 4$ and $\mathbf{S 5}$ ) were then investigated by SEM, revealing successful and selective NMOF positioning onto targeted areas of the substrate surface.

Figure 7 shows the data obtained by SEM imaging of the micro-patterned substrates. S4 (Figure $7 b-d$ ) is only partly covered, where the surface is functionalized with 1,8 -nonadiyne solution. This is further illustrated in Figure 7a. NMOF 2 binds to the areas with alkyne functionalization while the other areas are not covered. Another image, where this can be observed, can be found in the Supporting Information, (a) 54
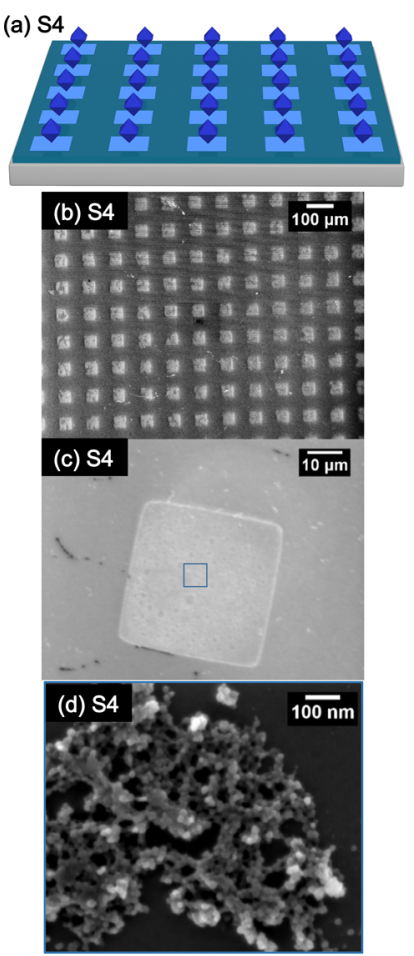

(e) S5
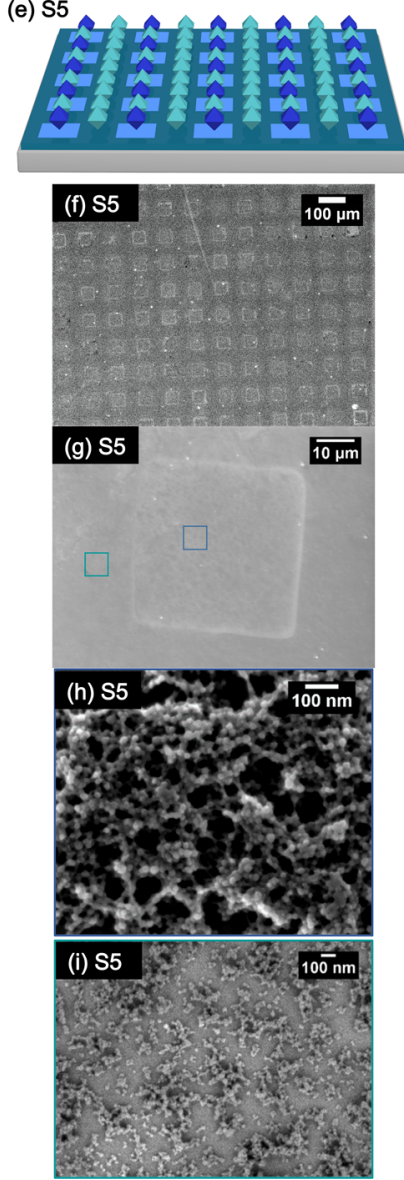

Figure 7. Selective NMOF positioning on micro-patterned substrates $(20 \times 20 \mu \mathrm{m})$ and SEM imaging. (a) Schematic representation of NMOF 2 (blue spheres) at the micro-patterned alkyne- (light blue) and carboxylic acid (dark turquoise)-terminated surface $\mathbf{S 4}$ and (e) NMOF 2 and NMOF 4 (turquoise spheres) at a similarly patterned surface S5, as well as the corresponding SEM images of the patterned Si surface S4 $(b-d)$ and S5 $(\mathrm{f}-\mathrm{i})$ after the reaction with NMOF 2 and NMOFs 2 and 4, respectively. The images show the surfaces in different magnifications. The blue and turquoise framed images are magnifications of the colored areas in the images (c) and (g), respectively. An image of the patterned surface without any NMOF can be found in the Supporting Information, Chapter S12.

Chapter S12. On the other hand, the surface S5 (Figure $7 \mathrm{f}-$ i) is fully covered with both NMOFs. Focusing the SEM on both regions of the substrate reveals that each of them shows the respective immobilized NMOFs. Figure 7a,e shows the schematic representation of this approach. The NMOF 2 (dark blue) binds to the alkyne-terminated regions (light blue), while the backfilled carboxylic acid-terminated areas (dark turquoise) form amide bonds with NMOF 4 (light turquoise). Addition- 
(a)
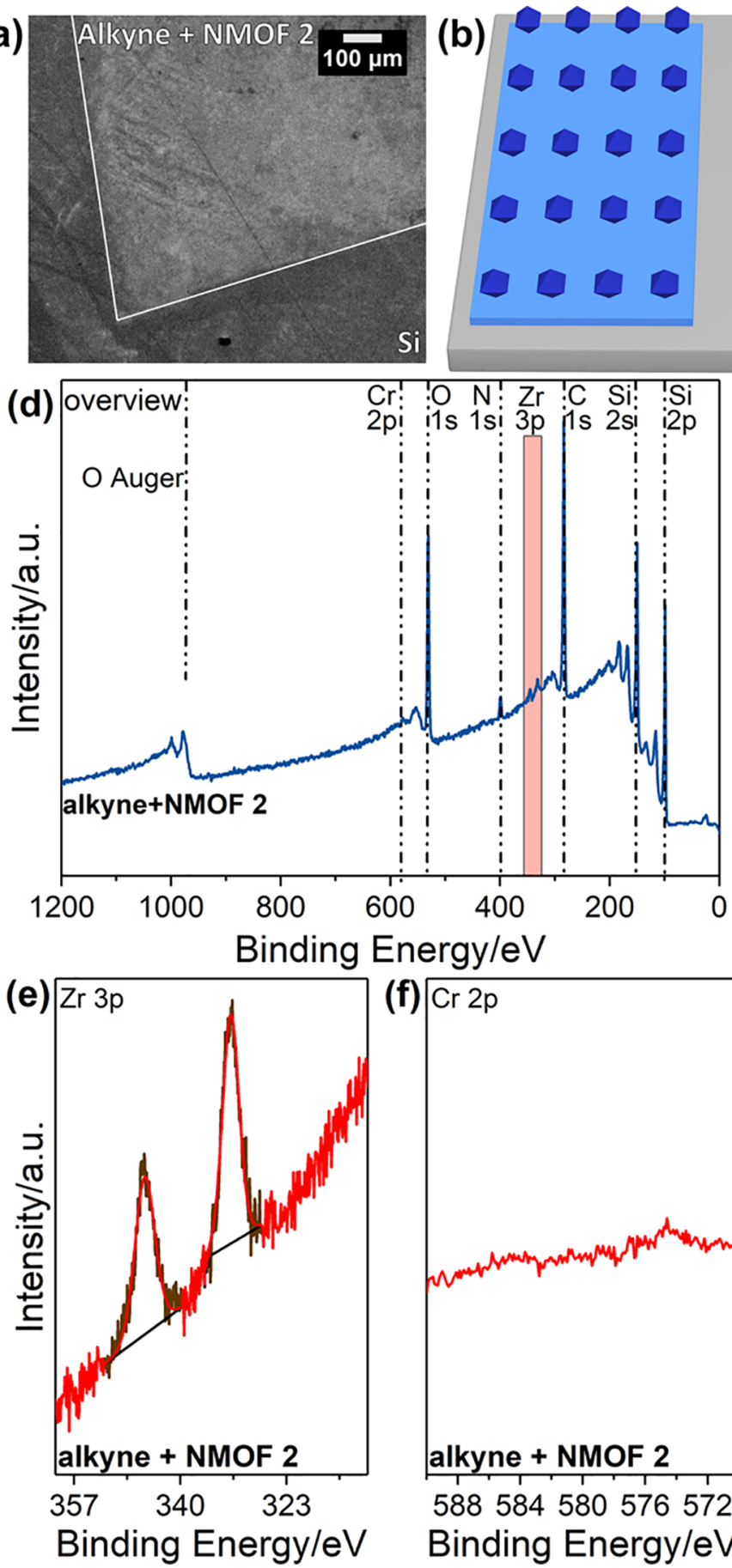

(b)

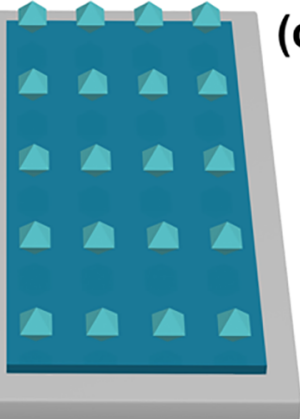

(c) Acid + NMOF 4
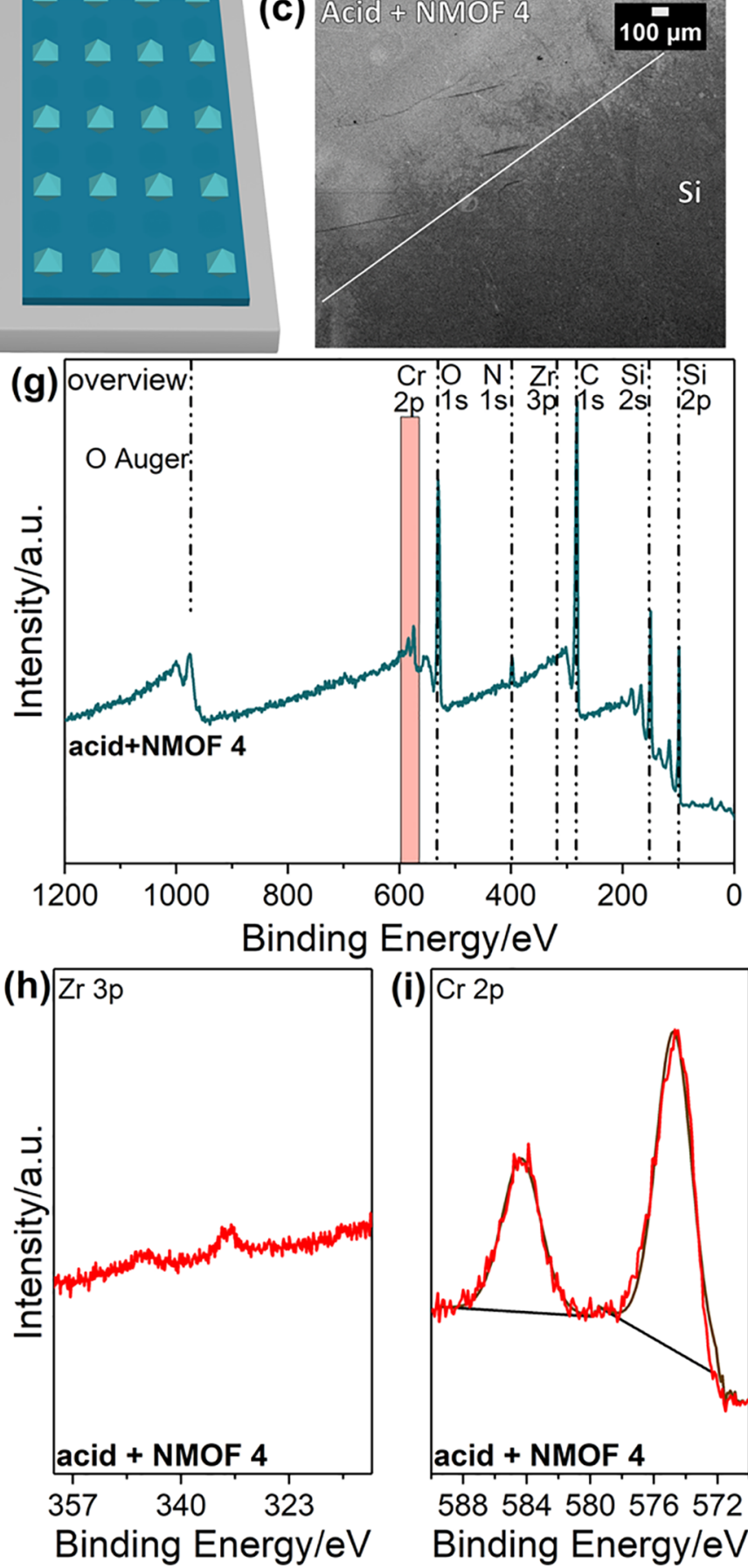

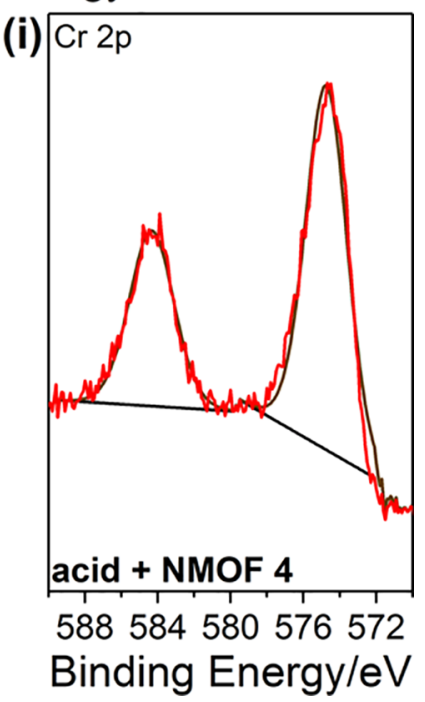

Figure 8. Selective NMOF positioning on macro-patterned substrates. Schematic representation of a silicon surface with a functionalization pattern created with PDMS substrates of $0.35 \times 0.4 \mathrm{~cm}$ dimension. The left side of the substrate was functionalized with 1,8-nonadiyne (light blue), while the right side was functionalized with 10-undecenoic acid (dark turquoise). After consecutive reactions with NMOF 2 (dark blue) and NMOF 4 (light turquoise), the substrate (a) was obtained and investigated by SEM (a,c) and XPS (d-i). SEM imaging (a,c) shows the macro-pattern in contrast to the silicon surface. For better clarification, the areas are marked with a white line. The raw images and more images can be found in the Supporting Information, Chapter S12. The survey spectra (d,g) and the $\operatorname{Zr~} 3 p(e, h)$ and the $\operatorname{Cr} 2 p(f, i)$ narrow scan were recorded for the alkyneterminated side (blue, $d-f$ ) and the acid-functionalized side (turquoise, $h-i)$, respectively.

ally, two sets of control experiments were conducted to elucidate the nature of the selective NMOF-to-surface binding. Specifically, when an alkyne-terminated surface is reacted with NMOF 4 and the acid-terminated surface is reacted with NMOF 2, no NMOFs were anchored to the substrate (see Supporting Information, Chapter S13). This proves that the NMOFs only stick to the substrates in the case of matching chemistry between the substrate and NMOF surfaces allowing for covalently binding NMOFs to the respective substrate surface. Unfortunately, we could not record meaningful SEMEDX images because of the large penetration depth of the method, only revealing the silicon surface underneath.

Instead, the selective positioning (spatial distribution) of the NMOFs on the functionalized surfaces was further investigated 
by SEM and XPS (Figure 8). To enable XPS focusing, we created an analogous macro-patterned surface. Both sides of the substrate $(1.0 \times 1.0 \mathrm{~cm})$ were functionalized with small PDMS substrates $(0.35 \times 0.4 \mathrm{~cm})$, creating two functionalized areas of $0.35 \times 0.4 \mathrm{~cm}$ each. Prior to the contact with the etched silicon surface, these PDMS substrates were immersed in either 1,8-nonadiyne or 10-undecanoic acid, which then created a functionalized macro-pattern on the silicon surface. After $12 \mathrm{~h}$ of contact between the PDMS substrate and the $\mathrm{Si}$ surface, the latter was washed and subsequentially reacted with first NMOF 2 (Figure 8a) and then NMOF 4 (Figure 8c). Finally, the surface was investigated by XPS using the characteristic $\mathrm{Zr}$ and $\mathrm{Cr}$ signals to identify the respective NMOFs. The analysis of the survey spectra, as well as the $\mathrm{Zr}$ $3 p$ and $\mathrm{Cr} 2 \mathrm{p}$ narrow scans of both parts of the substrates, clearly shows that NMOF $2(\mathrm{Zr})$ is only present on one side, where NMOF $4(\mathrm{Cr})$ is absent (Figure 8e,f). On the other side, however, NMOF $2(\mathrm{Zr})$ is absent and NMOF $4(\mathrm{Cr})$ is present (Figure $8 \mathrm{~h}, \mathrm{i}$ ). This provides unequivocal evidence of selective positioning of the NMOFs on the macro-patterned surface. It is reasonable to conclude that the same situation holds for the micropatterned substrates (Figure 7).

\section{CONCLUSIONS}

We investigated the immobilization of UiO-66 and MIL-101 NPs, which were functionalized on their exterior surface, on matching Si surfaces. Azide-terminated UiO-66 NPs were reacted with alkyne-functionalized Si surfaces, resulting in the triazole formation by click chemistry and their immobilization on the surface. Amino-functionalized MIL-101 NPs were combined with carboxylic acid-terminated Si surfaces yielding their covalent immobilization via amide bonds. Different characterization methods, for example, XPS and SEM, revealed the successful immobilization. Finally, a surface-patterning experiment demonstrated that the positioning of the NMOFs is selective and can be actively controlled by the choice of the pattern. Specifically, azide-functionalized NMOFs only interact with alkyne-terminated surfaces and amine-functionalized NMOFs only interact with carboxylic acid-terminated surfaces. This paves the way for the application of selective positioning of desired materials at targeted parts in microfluidic devices and further provides the possibility for selective, tandem, or cascade catalysis.

\section{EXPERIMENTAL SECTION}

Materials. Single side-polished silicon wafers (111) were purchased from Siegert Wafers and cut into $1 \times 1 \mathrm{~cm}^{2}$ pieces for further use.

NMOF Formation and Exterior Surface Functionalization. In a first step, UiO-66 (NMOF 1) and MIL-101 (NMOF 3) nanometersized crystals were synthesized, and in a second step, their exterior surface was functionalized by the formation of amide bonds to $\omega$ functionalized alkane- $\alpha$-amines to form azide-functionalized UiO-66 NPs (NMOF 2) and amine-functionalized MIL-101 (NMOF 4).

NMOF $1^{23}$ and 2 . A total of $21 \mathrm{mg}$ of zirconium oxychloride octahydrate $(0.066 \mathrm{mmol})$ and $50 \mathrm{mg}$ of benzene dicarboxylic acid ( $0.30 \mathrm{mmol}, 4.5$ equiv) were dissolved by sonication in $3 \mathrm{~mL}$ and 1 $\mathrm{mL}$ of dimethylformamide (DMF). Then, both solutions were mixed and $0.35 \mathrm{~mL}$ of acetic acid was added. Afterward, the reaction mixture was kept at $90{ }^{\circ} \mathrm{C}$ for $12 \mathrm{~h}$. The synthesis yields a white, colloidal solution. The solvent of the solution is exchanged by centrifugation three times with $4 \mathrm{~mL}$ of fresh DMF and three times with $4 \mathrm{~mL}$ of ethanol each. Then, $18 \mathrm{mg}$ of azidododecane-1-amine $(0.08 \mathrm{mmol})$ (synthesis procedure can be found in the Supporting Information,
Chapter S14), $22.3 \mu \mathrm{L}$ of $N, N$-diisopropylcarbodiimide (18 mg, 0.14 $\mathrm{mmol}$ ), and catalytic amounts of $\mathrm{N}$-hydroxysuccinimide were added to $4 \mathrm{~mL}$ of the colloidal NMOF 1 ethanol solution. After $4 \mathrm{~h}$ of stirring at room temperature, the reaction solution was exchanged three times replacing it with $4 \mathrm{~mL}$ of fresh ethanol each time. In between the centrifugation steps, the NMOF 2 solution was sonicated for several hours.

NMOF $3^{24}$ and 4. A total of $740.4 \mathrm{mg}$ of $\mathrm{Cr}\left(\mathrm{NO}_{3}\right)_{3} \cdot 9 \mathrm{H}_{2} \mathrm{O}(1.85$ mmol, 1.0 equiv) and $307.4 \mathrm{mg}$ of benzene dicarboxylic acid (1.85 mmol, 1.0 equiv) were dispersed in $10 \mathrm{~mL}$ of deionized water in a 50 $\mathrm{mL}$ microwave tube. After $10 \mathrm{~min}$ of stirring at room temperature, the reaction solution was heated with the maximum power to a set temperature of $200{ }^{\circ} \mathrm{C}$. After about $7 \mathrm{~min}$, the temperature reached $190^{\circ} \mathrm{C}$ and was kept at that temperature for $1 \mathrm{~min}$. After the reaction mixture was cooled down to room temperature, it was filtered over a Schott filter no. 2 to filter off unreacted benzene dicarboxylic acid. The colloidal green solution of NMOF 3 was centrifuged and the solution was replaced three times with fresh water $(20 \mathrm{~mL}$ each $)$ and three times with fresh ethanol $(20 \mathrm{~mL}$ each). A total of $20 \mathrm{~mL}$ of the green, colloidal NMOF 3 ethanol solution was mixed with $111.7 \mu \mathrm{L}$ of diisoproylcarbodiimide $(90 \mathrm{mg}, 0.71 \mathrm{mmol}), 180 \mathrm{mg}$ of DAD (0.90 mmol), and catalytic amounts of $\mathrm{N}$-hydroxysuccinimide and stirred for $4 \mathrm{~h}$ at room temperature. The solvent was exchanged three times after the reaction with $20 \mathrm{~mL}$ of ethanol each. In between the centrifugation, the NMOF 4 solution was sonicated for several hours.

Surface Preparation. The silicon wafers were cut into $1.0 \mathrm{~cm} \times$ $1.0 \mathrm{~cm}$ pieces. Prior to reaction, the silicon surfaces were cleaned by sonication in acetone, ethanol, and DCM for 5 min each. Afterward, they were dried under argon and activated with an oxygen plasma cleaner for $15 \mathrm{~min}$. In order to remove the native oxide on the silicon surface, the cleaned and activated Si surfaces were then treated for 30 min in degassed $40 \% \mathrm{NH}_{4} \mathrm{~F}$ solution. The substrates were blow-dried and kept under argon for further use.

Assembly of a 1,8-Nonadiyne Monolayer and CuAAC/Click Reaction. Hydrogen-terminated $\mathrm{Si}(\mathrm{Si}-\mathrm{H})$ substrates were placed in degassed 1,8-nonadiyne and left for $12 \mathrm{~h}$ at $100{ }^{\circ} \mathrm{C}$ under an argon atmosphere. After cooling to room temperature, the functionalized surface was rinsed copiously with water and sonicated in hexane and DCM for $5 \mathrm{~min}$ each. The blow-dried, alkyne-functionalized $\mathrm{Si}$ surfaces were reacted with NMOF 2 by CuAAC. Thereto, $6.5 \mathrm{mg}$ of copper (I) bromide tris(triphenylphosphine) $(7.0 \mu \mathrm{mol})$ and $0.5 \mathrm{~mL}$ of triethylamine were dissolved in $1 \mathrm{~mL}$ of THF. To this mixture, 1 $\mathrm{mL}$ of NMOF 2 ethanol solution and the alkyne-functionalized Si substrates were added and left at room temperature overnight, forming 2@Si (S1). Another approach that we tried was the CuAAC reaction in the microwave which also yielded good results. Therefore, $6.5 \mathrm{mg}$ of $\mathrm{CuSO}_{4} \cdot 5 \mathrm{H}_{2} \mathrm{O}(26 \mu \mathrm{mol})$ and $5 \mathrm{mg}$ of ascorbic acid $(28$ $\mu \mathrm{mol}$ ) were dissolved in $5 \mathrm{~mL}$ of deionized water and $1 \mathrm{~mL}$ of colloidal NMOF 2 in EtOH was added. After sonicating for $5 \mathrm{~min}$, the alkyne-functionalized substrate was added, and the reaction mixture was heated to $50{ }^{\circ} \mathrm{C}$ at $50 \mathrm{~W}$ for $30 \mathrm{~min}$. After the reaction, the substrate was rinsed with copious amounts of water and ethanol and sonicated with ethanol for $5 \mathrm{~min}$. After sonication in ethanol, the substrates were washed with DCM and blow-dried with argon.

Assembly of a 10-Undecenoic Acid Monolayer and Amide Formation. $\mathrm{Si}-\mathrm{H}$ substrates were placed in the degassed 10undecenoic acid and left for $12 \mathrm{~h}$ at $100{ }^{\circ} \mathrm{C}$ under an argon atmosphere. After cooling to room temperature, the functionalized surface was rinsed with water and sonicated in hexane and DCM for 5 min each. The blow-dried, acid-functionalized Si surfaces were reacted with NMOF 4 by NHS-catalyzed amide formation. Therefore, 23.3 $\mu \mathrm{L}$ of diisopropylcarbodiimide $(3.5 \mathrm{mmol})$ and catalytic amounts of $N$-hydroxysuccinimide were dissolved in $1 \mathrm{~mL}$ of ethanol. To this mixture, $3 \mathrm{~mL}$ of NMOF 4 ethanol solution and the acidfunctionalized $\mathrm{Si}$ substrate were added and reacted for $12 \mathrm{~h}$ at room temperature. After the reaction, the substrate $[4 @$ @i (S3)] was rinsed with copious amounts of water and ethanol and sonicated with ethanol for $5 \mathrm{~min}$. After sonication in ethanol, the substrates were washed with DCM and blow-dried with argon. 
Assembly of a 10-Undecenoic Acid/1,8-Nonadiyne Mixed Monolayer. The assembly of the mixed monolayer of a $1 / 1(n / n)$ solution of 10-undecenoic acid and 1,8-nonadiyne is prepared and degassed. The $\mathrm{Si}-\mathrm{H}$ surfaces are immersed in the solution under argon for $12 \mathrm{~h}$ at $100{ }^{\circ} \mathrm{C}$. Consecutively, after cooling to room temperature, the functionalized surface was rinsed with water and sonicated in hexane and DCM for 5 min each. In the next step, the surface was reacted with NMOF 2 in CuAAC and with NMOF 4 via amide coupling as described before, forming S2.

Microcontact Printing. To prepare the PDMS stamps, Sylgard 184 Silicone Elastomer Base and Sylgard 184 Silicone Curing Agent $(10 / 1 \mathrm{w} / \mathrm{w})$ were mixed for $3 \mathrm{~min}$, followed by degassing in a desiccator. The mixture is poured onto a mold and cured at $70^{\circ} \mathrm{C}$ for $3 \mathrm{~h}$. The stamps consist of rectangular islands of $20 \times 20 \mu \mathrm{m}$ diameter. For the patterning, $\mathrm{Si}-\mathrm{H}$ surfaces were transferred to a glovebox. The degassed 1,8-nonadiyne was dripped on a glass slide and the PDMS stamp was carefully placed on it. The 1,8-nonadiynesoaked PDMS stamp was then placed on a silicon substrate overnight. Afterward, the PDMS stamp was removed and the surface was cleaned by a rinse with hexane and DCM. For the backfilling, a drop of 10undecenoic acid was placed on the silicon substrate for $12 \mathrm{~h}$. After this time, the surface was rinsed with ethanol and sonicated in hexane and DCM. We also inverted the experiment, with 10 -undecenoic acid in the microcontact printing step and 1,8-nonadiyne in the backfilling step (see Supporting Information Chapter 12). This surface was successively reacted with NMOF 2 and $\mathbf{4}$ as described before, creating S4 and S5.

Further Patterning Experiments. PDMS substrates were cut into $0.35 \times 0.4 \mathrm{~cm}$ pieces, immersed in either 1,8 -nonadiyne or 10 undecenoic acid for $5 \mathrm{~min}$ and subsequently placed on an etched silicon substrate under inert conditions (glovebox). Afterward, the PDMS stamps were removed and the surface was cleaned by sonication with ethanol, hexane, and DCM, for 5 min each. Finally, the substrate was successively reacted with NMOF 2 and 4 as described before.

\section{ASSOCIATED CONTENT}

\section{(s) Supporting Information}

The Supporting Information is available free of charge at https://pubs.acs.org/doi/10.1021/acs.chemmater.0c02871.

General information about instrumentation, detailed information about performed reactions, SEM and contact angle images, IRRAS, NMR, and XPS spectra, $\mathrm{N}_{2}$ adsorption isotherms, and detailed instruction regarding the performed calculations (PDF)

\section{AUTHOR INFORMATION}

\section{Corresponding Author}

Roland A. Fischer - Chair of Inorganic and Metal-Organic Chemistry, Department of Chemistry, Technical University of Munich, Garching 85748, Germany; 이잉.org/00000002-7532-5286; Phone: +49-89-289-13081; Email: roland.fischer@tum.de; Fax: +49-89-289-13194

\section{Authors}

Anna Lisa Semrau - Chair of Inorganic and Metal-Organic Chemistry, Department of Chemistry, Technical University of Munich, Garching 85748, Germany; 이이이.org/00000001-7087-932X

Sidharam P. Pujari - Laboratory of Organic Chemistry, Wageningen University, Wageningen 6708, The Netherlands; (1) orcid.org/0000-0003-0479-8884

Philip Matthew Stanley - Chair of Inorganic and Metal-Organic Chemistry, Department of Chemistry, Technical University of Munich, Garching 85748, Germany; (1) orcid.org/0000-0002-1951-4074
Suttipong Wannapaiboon - Synchrotron Light Research Institute, Nakhon Ratchasima 30000, Thailand

Bauke Albada - Laboratory of Organic Chemistry, Wageningen University, Wageningen 6708, The Netherlands; (1) orcid.org/0000-0003-3659-2434

Han Zuilhof - Laboratory of Organic Chemistry, Wageningen University, Wageningen 6708, The Netherlands; School of Pharmaceutical Sciences and Technology, Tianjin University, Tianjin 300072, P. R. China; Department of Chemical and Materials Engineering, Faculty of Engineering, King Abdulaziz University, Jeddah 23218, Saudi Arabia; (1) orcid.org/0000-0001-5773-8506

Complete contact information is available at: https://pubs.acs.org/10.1021/acs.chemmater.0c02871

\section{Author Contributions}

The manuscript was written through contributions of all authors. All authors have given approval to the final version of the manuscript.

Notes

The authors declare no competing financial interest.

\section{ACKNOWLEDGMENTS}

A.L.S. and P.M.S. are grateful for a PhD scholarship from the Chemical Industry Fund (FCI). This work was supported by the German Research Foundation (DFG) Priority Program 1928 "Coordination Networks: Building Blocks for Functional Systems". A.L.S. is grateful for lab space, access to the analytical instruments, and help from staff and group members in the MPI for Solid State Physics in the group from Bettina Lotsch from March-May 2018. We would like to acknowledge the help of Katja Rodewald for measuring SEM images. Additionally, we would like to thank the group from Prof. Hubert Gaststeiger for allowing us access to their XPS instrument and for Christian Jandl for conducting a part of the XPS measurements presented in this manuscript.

\section{REFERENCES}

(1) Gao, W.; Zhang, Z.; Li, J.; Ma, Y.; Qu, Y. Surface Engineering on $\mathrm{CeO}_{2}$ Nanorods by Chemical Redox Etching and Their Enhanced Catalytic Activity for CO Oxidation. Nanoscale 2015, 7, 1168611691.

(2) Li, Y.-Y.; Si, Y.; Zhou, B.-X.; Huang, W.-Q.; Hu, W.; Pan, A.; Fan, X.; Huang, G.-F. Strategy to Boost Catalytic Activity of Polymeric Carbon Nitride: Synergistic Effect of Controllable: In Situ Surface Engineering and Morphology. Nanoscale 2019, 11, 16393-16405.

(3) Yetisen, A. K.; Butt, H.; Volpatti, L. R.; Pavlichenko, I.; Humar, M.; Kwok, S. J. J.; Koo, H.; Kim, K. S.; Naydenova, I.; Khademhosseini, A.; Hahn, S. K.; Yun, S. H. Photonic Hydrogel Sensors. Biotechnol. Adv. 2016, 34, 250-271.

(4) Dohnalová, K.; Poddubny, A. N.; Prokofiev, A. A.; De Boer, W. D.; Umesh, C. P.; Paulusse, J. M.; Zuilhof, H.; Gregorkiewicz, T. Surface Brightens up Si Quantum Dots: Direct Bandgap-like SizeTunable Emission. Light Sci. Appl. 2013, 2, No. e47.

(5) Batchelor, A. W.; Lam, L. N.; Chandrasekaran, M. Materials Degradation and Its Control by Surface Engineering; Imperial College Press: London, 2011.

(6) Kitagawa, S.; Kitaura, R.; Noro, S.-i. Functional Porous Coordination Polymers. Angew. Chem., Int. Ed. 2004, 43, 2334-2375.

(7) Li, H.; Wang, K.; Sun, Y.; Lollar, C. T.; Li, J.; Zhou, H.-C. Recent Advances in Gas Storage and Separation Using MetalOrganic Frameworks. Mater. Today 2018, 21, 108-121. 
(8) Bavykina, A.; Kolobov, N.; Khan, I. S.; Bau, J. A.; Ramirez, A.; Gascon, J. Metal-Organic Frameworks in Heterogeneous Catalysis: Recent Progress, New Trends, and Future Perspectives. Chem. Rev. 2020, 120, 8468-8535.

(9) Liu, Y.; Xie, X.-Y.; Cheng, C.; Shao, Z.-S.; Wang, H.-S. Strategies to Fabricate Metal-Organic Framework (MOF)-Based Luminescent Sensing Platforms. J. Mater. Chem. C 2019, 7, 10743-10763.

(10) Bilal, M.; Adeel, M.; Rasheed, T.; Iqbal, H. M. N. Multifunctional Metal-Organic Frameworks-Based Biocatalytic Platforms: Recent Developments and Future Prospects. J. Mater. Res. Technol. 2019, 8, 2359-2371.

(11) Multilayer Thin Films-Versatile Applications for Materials Engineering; Basu, S., Ed.; IntechOpen: London, 2020.

(12) Munuera, C.; Shekhah, O.; Wang, H.; Wöll, C.; Ocal, C. The Controlled Growth of Oriented Metal-Organic Frameworks on Functionalized Surfaces as Followed by Scanning Force Microscopy. Phys. Chem. Chem. Phys. 2008, 10, 7257.

(13) Falcaro, P.; Hill, A. J.; Nairn, K. M.; Jasieniak, J.; Mardel, J. I.; Bastow, T. J.; Mayo, S. C.; Gimona, M.; Gomez, D.; Whitfield, H. J.; Riccò, R.; Patelli, A.; Marmiroli, B.; Amenitsch, H.; Colson, T.; Villanova, L.; et al. A New Method to Position and Functionalize Metal-Organic Framework Crystals. Nat. Commun. 2011, 2, 237.

(14) Falcaro, P.; Ricco, R.; Doherty, C. M.; Liang, K.; Hill, A. J.; Styles, M. J. MOF Positioning Technology and Device Fabrication. Chem. Soc. Rev. 2014, 43, 5513-5560.

(15) Li, P.; Klet, R. C.; Moon, S.-Y.; Wang, T. C.; Deria, P.; Peters, A. W.; Klahr, B. M.; Park, H.-J.; Al-Juaid, S. S.; Hupp, J. T. Synthesis of Nanocrystals of Zr-Based Metal-Organic Frameworks with CsqNet: Significant Enhancement in the Degradation of a Nerve Agent Simulant. Chem. Commun. 2015, 51, 10925-10928.

(16) Herbst, A.; Khutia, A.; Janiak, C. Brønsted Instead of Lewis Acidity in Functionalized MIL-101Cr MOFs for Efficient Heterogeneous (Nano-MOF) Catalysis in the Condensation Reaction of Aldehydes with Alcohols. Inorg. Chem. 2014, 53, 7319-7333.

(17) Semrau, A. L.; Stanley, P. M.; Urstoeger, A.; Schuster, M.; Cokoja, M.; Fischer, R. A. Substantial Turnover Frequency Enhancement of MOF Catalysts by Crystallite Downsizing Combined with Surface Anchoring. ACS Catal. 2020, 10, 3203-3211.

(18) Cavka, J. H.; Jakobsen, S.; Olsbye, U.; Guillou, N.; Lamberti, C.; Bordiga, S.; Lillerud, K. P. A New Zirconium Inorganic Building Brick Forming Metal Organic Frameworks with Exceptional Stability. J. Am. Chem. Soc. 2008, 130, 13850-13851.

(19) Férey, G.; Serre, C.; Mellot-Draznieks, C.; Millange, F.; Surblé, S.; Dutour, J.; Margiolaki, I. A Hybrid Solid with Giant Pores Prepared by a Combination of Targeted Chemistry, Simulation, and Powder Diffraction. Angew. Chem., Int. Ed. 2004, 43, 6296-6301.

(20) Mondloch, J. E.; Katz, M. J.; Planas, N.; Semrouni, D.; Gagliardi, L.; Hupp, J. T.; Farha, O. K. Are $\mathrm{Zr}_{6}$-Based MOFs Water Stable? Linker Hydrolysis vs. Capillary-Force-Driven Channel Collapse. Chem. Commun. 2014, 50, 8944-8946.

(21) DeCoste, J. B.; Peterson, G. W.; Jasuja, H.; Glover, T. G.; Huang, Y.-g.; Walton, K. S. Stability and Degradation Mechanisms of Metal-Organic Frameworks Containing the $\mathrm{Zr}_{6} \mathrm{O}_{4}(\mathrm{OH})_{4}$ Secondary Building Unit. J. Mater. Chem. A 2013, 1, 5642.

(22) Bhattacharjee, S.; Chen, C.; Ahn, W.-S. Chromium Terephthalate Metal-Organic Framework MIL-101: Synthesis, Functionalization, and Applications for Adsorption and Catalysis. RSC Adv. 2014, 4, 52500-52525.

(23) Wang, S.; Morris, W.; Liu, Y.; McGuirk, C. M.; Zhou, Y.; Hupp, J. T.; Farha, O. K.; Mirkin, C. A. Surface-Specific Functionalization of Nanoscale Metal-Organic Frameworks. Angew. Chem., Int. Ed. 2015, 54, 14738-14742.

(24) Demessence, A.; Horcajada, P.; Serre, C.; Boissière, C.; Grosso, D.; Sanchez, C.; Férey, G. Elaboration and Properties of Hierarchically Structured Optical Thin Films of MIL-101(Cr). Chem. Commun. 2009, 7149-7151.

(25) Coffinier, Y.; Piret, G.; Das, M. R.; Boukherroub, R. Effect of Surface Roughness and Chemical Composition on the Wetting
Properties of Silicon-Based Substrates. Compt. Rendus Chem. 2013, $16,65-72$.

(26) Wang, J.; Wu, F.; Watkinson, M.; Zhu, J.; Krause, S. "Click" Patterning of Self-Assembled Monolayers on Hydrogen-Terminated Silicon Surfaces and Their Characterization Using Light-Addressable Potentiometric Sensors. Langmuir 2015, 31, 9646-9654.

(27) Ciampi, S.; Böcking, T.; Kilian, K. A.; James, M.; Harper, J. B.; Gooding, J. J. Functionalization of Acetylene-Terminated Monolayers on $\mathrm{Si}(100)$ Surfaces: A Click Chemistry Approach. Langmuir 2007, 23, 9320-9329.

(28) Liu, H.; Duclairoir, F.; Fleury, B.; Dubois, L.; Chenavier, Y.; Marchon, J.-C. Porphyrin Anchoring on $\mathrm{Si}(100)$ Using a $\beta$-Pyrrolic Position. Dalton Trans. 2009, 3793-3799.

(29) Voicu, R.; Boukherroub, R.; Bartzoka, V.; Ward, T.; Wojtyk, J. T. C.; Wayner, D. D. M. Formation, Characterization, and Chemistry of Undecanoic Acid-Terminated Silicon Surfaces: Patterning and Immobilization of DNA. Langmuir 2004, 20, 11713-11720.

(30) Alvarez, S. D.; Derfus, A. M.; Schwartz, M. P.; Bhatia, S. N.; Sailor, M. J. The Compatibility of Hepatocytes with Chemically Modified Porous Silicon with Reference to in Vitro Biosensors. Biomaterials 2009, 30, 26-34.

(31) Marshall, C. R.; Timmel, E. E.; Staudhammer, S. A.; Brozek, C. K. Experimental Evidence for a General Model of Modulated MOF Nanoparticle Growth. Chem. Sci. 2020, 11, 11539.

(32) Howarth, A. J.; Peters, A. W.; Vermeulen, N. A.; Wang, T. C.; Hupp, J. T.; Farha, O. K. Best Practices for the Synthesis, Activation, and Characterization of Metal-Organic Frameworks. Chem. Mater. 2017, 29, 26-39.

(33) Lange, S. C.; van Andel, E.; Smulders, M. M. J.; Zuilhof, H. Efficient and Tunable Three-Dimensional Functionalization of Fully Zwitterionic Antifouling Surface Coatings. Langmuir 2016, 32, 10199-10205.

(34) Zhao, J.; Gao, F.; Pujari, S. P.; Zuilhof, H.; Teplyakov, A. V. Universal Calibration of Computationally Predicted N 1s Binding Energies for Interpretation of XPS Experimental Measurements. Langmuir 2017, 33, 10792-10799. 